\title{
Scanning Gate Microscope for Cold Atomic Gases
}

\author{
Samuel Häusler, ${ }^{1, *}$ Shuta Nakajima, ${ }^{2}$ Martin Lebrat, ${ }^{1}$ Dominik Husmann, ${ }^{1}$ Sebastian Krinner, ${ }^{1}$ \\ Tilman Esslinger, ${ }^{1, \dagger}$ and Jean-Philippe Brantut, ${ }^{3, \$}$ \\ ${ }^{1}$ Department of Physics, ETH Zurich, 8093 Zurich, Switzerland \\ ${ }^{2}$ Department of Physics, Graduate School of Science, Kyoto University, Kyoto 606-8502, Japan \\ ${ }^{3}$ Institute of Physics, EPFL, 1015 Lausanne, Switzerland
}

(Received 8 February 2017; published 21 July 2017)

\begin{abstract}
We present a scanning probe microscopy technique for spatially resolving transport in cold atomic gases, in close analogy with scanning gate microscopy in semiconductor physics. The conductance of a quantum point contact connected to two atomic reservoirs is measured in the presence of a tightly focused laser beam acting as a local perturbation that can be precisely positioned in space. By scanning its position and recording the subsequent variations of conductance, we retrieve a high-resolution map of transport through a quantum point contact. We demonstrate a spatial resolution comparable to the extent of the transverse wave function of the atoms inside the channel and a position sensitivity below $10 \mathrm{~nm}$. Our measurements agree well with an analytical model and $a b$ initio numerical simulations, allowing us to identify a regime in transport where tunneling dominates over thermal effects. Our technique opens new perspectives for the high-resolution observation and manipulation of cold atomic gases.
\end{abstract}

DOI: 10.1103/PhysRevLett.119.030403

Scanning probe microscopes had a substantial impact on the development of solid-state physics during the past three decades, from the observation of individual atoms at surfaces [1,2] to the imaging of coherent electron flow [3] and the identification of order parameters in complex correlated materials $[4,5]$ - just to name a few examples. Many of these applications rely on two conceptually important ingredients: (i) the use of very sharp probes positioned with atomic-scale precision close to a surface and (ii) the ability to continuously measure transport in the presence of a local probe, which yields precise information related to a single point of the system by accumulating the often weak transport signal.

Many fundamental phenomena observed in condensed matter physics are also studied in cold-atom-based quantum simulations [6]. This has motivated the development of high spatial resolution imaging based on photon [7-16] and electron [17] scattering, which typically yields a destructive observation of the local density distribution or the parity of the atom number on lattice sites. Yet, a high spatial resolution measurement in a transport setting, as has been successfully applied in solid-state physics, has so far not been carried out with atomic gases.

In this Letter, we demonstrate a scanning gate microscope for a cold atomic gas flowing through an optically created quantum point contact (QPC) [18]. Our technique is inspired by scanning gate microscopy in semiconductor physics [3,19-21], where a movable gate potential is used to locally modify the underlying carrier density in a sample. In our cold-atom implementation, we use a high-resolution optical microscope to create a submicrometer repulsive gate potential in the region of the QPC. Thanks to the intrinsic diluteness of cold atomic gases, our gate operates at the scale of the Fermi wavelength.

Our technique complements direct fluorescence or absorption imaging in many respects. At the conceptual level, it uses quantum degenerate atoms themselves, rather than photons, as test particles incident on the system [22]. Large reservoirs connected to a smaller, mesoscopic system act as the source and sink for the scattered atoms, continuously accumulating the signal. Since no spontaneous emission of photons or other dissipative processes are induced during the accumulation, it is possible to access long time scales. In contrast, photon or electron scattering provides an instantaneous snapshot of the density distribution.

The basis of our experiment is a quantum degenerate Fermi gas of ${ }^{6} \mathrm{Li}$ atoms, as described in our previous work [18]. The Fermi gas is produced in a combined magnetic and optical trap, yielding an elongated cloud with $N=$ $1.01(7) \times 10^{5}$ atoms in each of the lowest and third-lowest hyperfine states of lithium. A homogeneous magnetic field of $949 \mathrm{G}$ is applied, which sets the scattering length to $-2653 a_{0}$, where $a_{0}$ is Bohr's radius. This corresponds to an interaction parameter $1 / k_{F} a$ in the reservoirs of -2.1 , where $k_{F}=\sqrt{2 m E_{F}} / \hbar$ is the Fermi wave vector in the gas, $m$ is the mass of lithium atoms, and $E_{F}=\hbar \bar{\omega}(6 N)^{1 / 3}$ is the Fermi energy in the harmonic trap, with $\bar{\omega}$ the geometric mean of its frequencies. At typical temperatures of about $60 \mathrm{nK}$, we expect our gas to be in the normal phase, as the critical temperature for superfluidity is $42 \mathrm{nK}$ [23]. As presented in Fig. 1(a), a repulsive potential generated by a laser beam with a nodal line in the middle is imposed on the cloud, creating a quasi-two-dimensional Fermi gas at the center of the cloud, smoothly connected on both sides to 
(a)

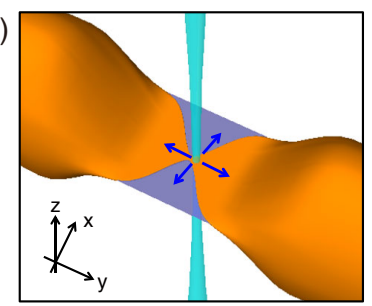

(b)

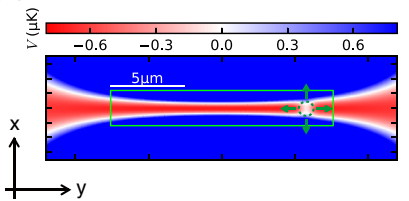

(c)

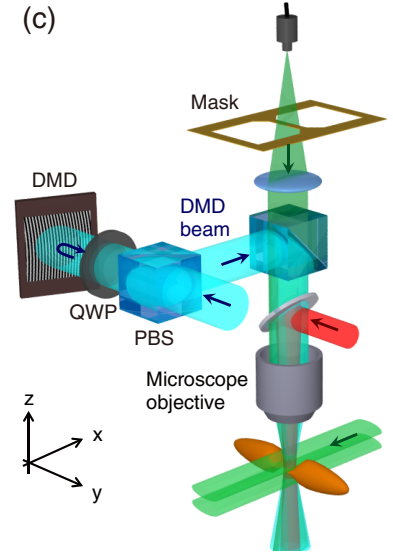

FIG. 1. An atomic scanning gate microscope. (a) Close-up view of the channel region: mesoscopic reservoirs, 2D regions, and the QPC. The scanning gate is realized by a tightly focused, repulsive laser beam that is scanned across the structure. (b) The gate (green circle) locally modifies the potential landscape shown for the parameters used in the simulation, Fig. 2(b). The green square indicates the region mapped in Fig. 2(c). Engineering of the QPC and the scanning gate. The QPC is formed at the intersection of two repulsive laser beams (indicated in green) having a nodal line at the center. $\mathrm{A} \mathrm{TEM}_{01}$-like laser mode propagating along the $-x$ axis forms the 2D regions. The beam passing through a lithographic mask is shrunk onto the $2 \mathrm{D}$ region and creates the QPC. The scanning gate is holographically shaped and moved (blue beam) by a DMD and projected onto the atoms with a microscope objective. The red beam creates an attractive potential to locally shift the chemical potential.

large, three-dimensional reservoirs [36]. The trap frequency (mode spacing) along the vertical $(z)$ direction at the center of the quasi-two-dimensional region reaches $\omega_{z}=2 \pi \times 13(5) \mathrm{kHz}$. The QPC is produced by imaging a binary mask using light at $532 \mathrm{~nm}$, imprinting a thin wire onto the quasi-two-dimensional region similar to Ref. [18]. We reach trap frequencies along the transverse direction of about $\omega_{x}=2 \pi \times 22(9) \mathrm{kHz}$ at the center of the QPC. Along the transport direction $(y)$, the beam producing the QPC has a waist of 9.1(3) $\mu \mathrm{m}$. An attractive potential produced by a Gaussian, red-detuned beam with a waist of $42.5(3) \mu \mathrm{m}$ is superimposed onto the QPC, allowing for the control of the chemical potential in the QPC and its immediate vicinity. Upon increasing the chemical potential, successive transverse modes of the QPC are populated yielding characteristic conductance plateaus [18].

We operate the QPC in the single-mode regime by tuning the chemical potential to the center of the first plateau. To obtain clear signals, we first work in the strong probe limit, where no theory unambiguously relates the response to the scanning gate to local observables. In contrast, weak probes directly yield a map of the current distribution, in the special case of a narrow tip probing a symmetric QPC in the single-mode regime [37]. The gate potential is produced by a tightly focused beam at $532 \mathrm{~nm}$ with waists

of $w_{x}=731(1) \mathrm{nm}$ and $w_{y}=751(1) \mathrm{nm}$. This beam is shaped and controlled using a digital mirror device (DMD), operating in the Fourier plane of the microscope as a diffraction grating in the Littrow configuration (Fig. 1 and Ref. [23]). We scan its position in the region indicated in Fig. 1(b) with an extent of $15.2 \mu \mathrm{m} \times 2.4 \mu \mathrm{m}$. The resulting map is shown in Fig. 2, where each pixel represents the conductance with the scanned gate at the position of the pixel. The individual measurements are separated by $238 \mathrm{~nm}$, above the minimal displacement of $93 \mathrm{~nm}$ set by the discreteness of the DMD and our optical setup. We use a gate strength of $V_{0}=620(1) \mathrm{nK} k_{B}$, about twice the local Fermi energy at the center of the structure, corresponding to the strong probe regime [39]. The region of low conductance represents the center of the QPC, where the current density is the highest. The pattern fades out towards the edges along $y$, where the current density is smaller due to the weaker confinements. Classically, these are regions where the extension of the gate is smaller than the width of the conductor. The full width at half maximum (FWHM) of the conductance pattern along the $y$ direction is 12(1) $\mu \mathrm{m}$, matching that of the beam creating the QPC. Along the transverse $(x)$ direction, the short FWHM of $0.8(2) \mu \mathrm{m}$ results from the tight confinement of the QPC.

We compare the experimental results with direct numerical simulations of the scanning gate setup using the Kwant library $[23,40]$. This solves the scattering problem of

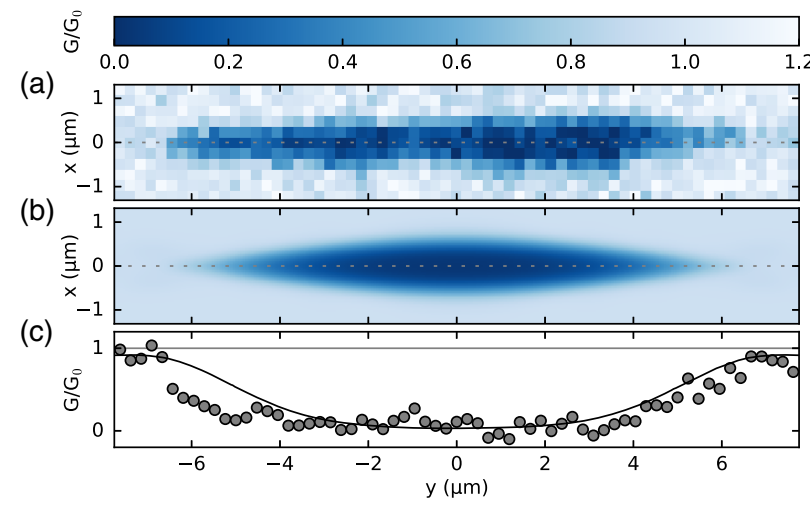

FIG. 2. Scanning gate map of a single-mode QPC. Measured (a) and calculated (b) conductance $G$ normalized to the background value $G_{0}$ as a function of the position of the scanning gate imposed onto the QPC. Because of the weak attraction among the particles, the measured background of $1.3 / h$ is larger than the universal conductance of $1 / h$ [38]. The scanning gate has a strength of $620(1) n K k_{B}$. The reservoir acting as the source is located on the left and the sink on the right side of the map. The simulation considers a single free parameter, the chemical potential in the reservoirs. The temperature and the chemical potential bias are $58(7) \mathrm{nK}$ and $99(4) \mathrm{nK} k_{B}$, respectively, calibrated independently [23]. (c) Longitudinal cuts through the scanning gate maps, along the dashed lines in (a) and (b). The measured conductances are represented by the points and the simulation by the line. 
independent particles originating from ideal reservoirs and impinging onto the structure. The potential landscape of the QPC along $x$ and $y$ is set a priori from the geometry of the laser beams, and the chemical potential is adjusted to fit the data. The results of the simulation are shown in Fig. 2(b), showing overall good agreement with the experiment. In particular, the transverse and longitudinal shapes are reproduced, as well as the fading out of the pattern in the wings of the QPC.

It was observed in the condensed matter context that scanning gate maps are dressed by fringe patterns, resulting from interferences between particles emitted by the point contact and reflected by the scanning gate [3]. In our experiment, these fringes are washed out by a finite temperature, as confirmed by our numerical simulations [23]. In contrast to semiconductor nanostructures, where large-scale disorder channels the particles emitted by the QPC [3], our system is free of disorder, and channeling does not take place.

We now study the regimes of scanning gate microscopy, from weak to strong probes. To this end, we scan the gate transversally through the center of the QPC, with varying $V_{0}$. These cuts are shown in Fig. 3. For the lowest $V_{0}$, the channel is not closed even with the scanning gate at the center of the QPC. This corresponds to the weak probe regime. As $V_{0}$ is increased, the conductance quickly goes to zero when the tip is at the center, and the profile changes from approximately Gaussian to flattop. For stronger scanning gates, the QPC is fully closed over an increasingly wide range, reflecting a clipping effect.

To analytically describe the scanning gate response, we model the QPC in the presence of the tip. The QPC tightly confines the particles laterally, such that they explore only a longitudinal cut through the Gaussian tip potential. This is justified, as the ground state wave function has a FWHM of $l=1.67 \sqrt{\hbar / m \omega_{x}}=0.5(2) \mu \mathrm{m}$ inside the QPC, about half that of the tip in the transverse direction. Furthermore, we approximate the longitudinal potential cut by a parabolic barrier with antitrapping frequency $\Omega(x)=$ $2 / w_{y} \sqrt{V(x) / m}$ and potential offset $V(x)=V_{0} e^{-2 x^{2} / w_{x}^{2}}$, where $x$ is the transverse tip position with respect to the QPC center. The transmission $\mathcal{T}$ through the parabolic barrier is given by

$$
\mathcal{T}(E)=\frac{1}{1+e^{-2 \pi \frac{E-V(x)}{\hbar \Omega(x)}}},
$$

where $E$ is the energy of the incident particle [41,42]. We combine the transmission $\mathcal{T}(E)$ with the thermal occupation of states in the reservoirs to obtain conductances, using Landauer's formula [42]. The profiles calculated using this model are shown in Fig. 3(c), where the overall chemical potential is the only free parameter common to all curves. The agreement with the measurement is good over the whole range of parameters, as can be seen on the cuts in
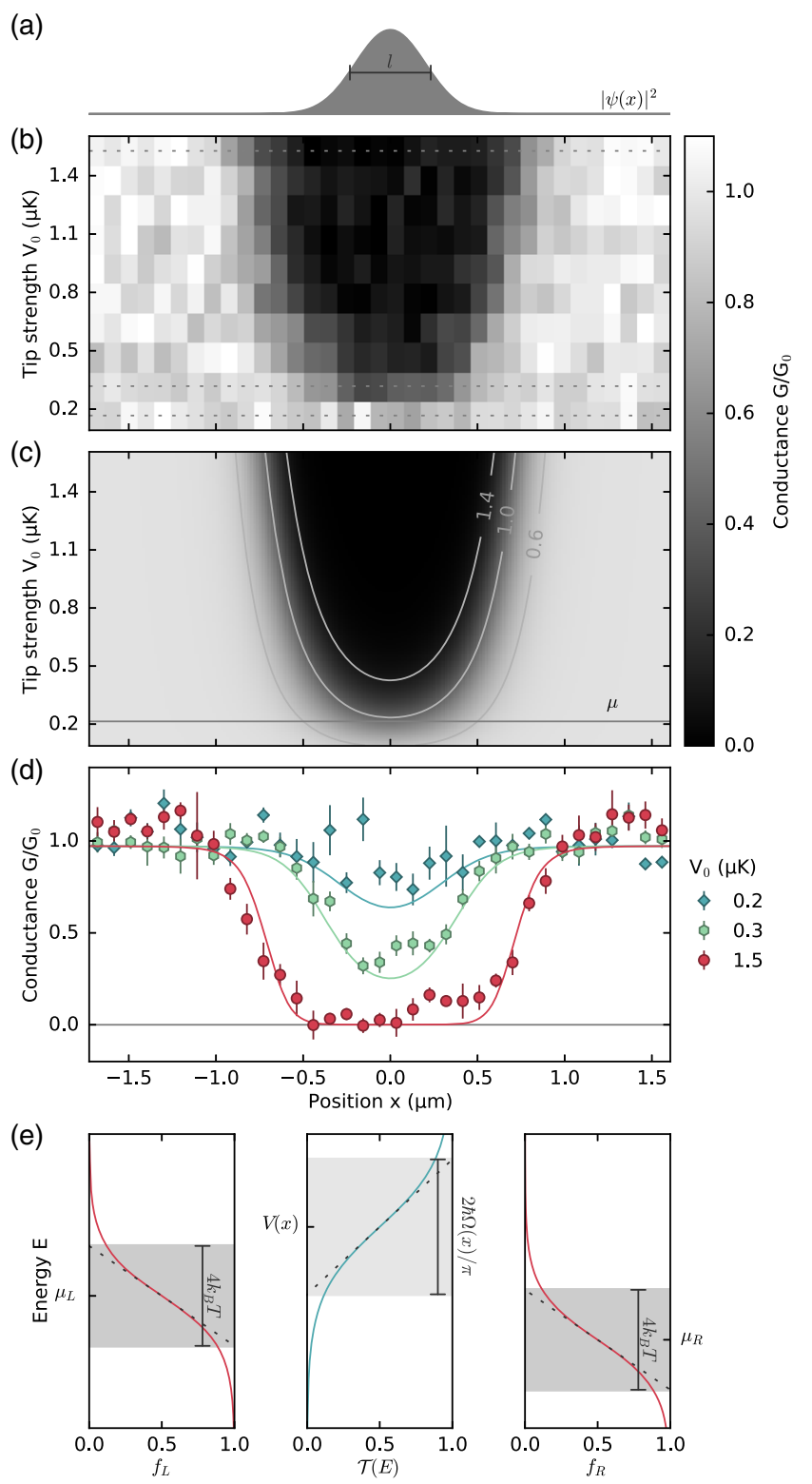

FIG. 3. Transverse scans through the QPC's center in the singlemode regime. (a) Transverse ground state probability distribution with FWHM $l$ of $0.5(2) \mu \mathrm{m}$ in the absence of the tip. (b) Conductance normalized to the measured background value $G_{0}$ of $1.1 / h$ as a function of the tip position $x$ and strength $V_{0}$. The dotted lines indicate representative cuts shown in (d). (c) Prediction by the analytical model including the chemical potential as the only free parameter, while the temperature and chemical potential bias are independently calibrated to $58(5) \mathrm{nK}$ and $102(3) \mathrm{nK} k_{B}$, respectively. The fitted chemical potential $\mu$ is indicated in the plot. Solid lines mark realizations with the same ratio $\hbar \Omega(x) / 2 \pi k_{B} T$. (d) Transverse scans for different tip strengths $V_{0}$. The solid curves indicate best fits with the analytical model. (e) Fermi-Dirac distribution $f_{L / R}(E)$ of each reservoir centered around the chemical potential $\mu_{L / R}$. They are smeared over the energy scale $4 k_{B} T$ (dark shaded area), defined by the tangential line (dashed line) at the inflection point. The transmission $\mathcal{T}(E)$ through a parabolic barrier with height $V(x)$ and the associated energy scale $2 \hbar \Omega(x) / \pi$ (light shaded area) is shown in the tunneling-dominated regime, for a ratio $\hbar \Omega(x) / 2 \pi k_{B} T$ of 1.4 (regime discussed in Ref. [23]). 
Fig. 3(d). We also compared this model with the numerically exact Kwant simulation, finding good agreement [23].

For a given position and strength of the tip, the analytical model allows us to compare the contributions of quantum tunneling through the tip and thermally activated particles passing over it. Since the transmission $\mathcal{T}(E)$ and the energy distribution are described by Fermi functions, the relative weight of the two is given by the ratio of the two energy scales $\hbar \Omega(x) / 2 \pi k_{B} T$ (explicit derivation in Ref. [23]). For strong tips, $2 \hbar \Omega(x) / \pi$ is larger than $4 k_{B} T$, as illustrated in Fig. 3(e), showing that the weak current mainly originates from tunneling [43]. The ratio $\hbar \Omega(x) / 2 \pi k_{B} T$ is presented as contour lines in Fig. 3(c), revealing that we can detect nonzero conductances with ratios up to 1.4 [23]. This is in contrast to experiments in condensed matter physics where direct tunneling through the scanned gate is negligible.

To estimate the resolution, we measure the FWHM of the transverse cuts of Fig. 3 [23]. The results are presented in Fig. 4(a), as a function of $V_{0}$. For strong gates, the FWHM is large, because the QPC is already blocked by the raising edges of the Gaussian gate. Reducing $V_{0}$, the FWHM goes down and becomes smaller than that of the laser beam for $V_{0}<0.4 \mu \mathrm{K}$. Interestingly, it keeps decreasing for lower $V_{0}$, suggesting that the resolution is not limited by the optical beam profile, similar to superresolved optical techniques reaching resolutions beyond the diffraction limit [44] as a consequence of the nonlinear transmission coefficient in Eq. (1). For the smallest $V_{0}$, the signal is weak, but the FWHM is low enough to be comparable with that of the transverse ground state wave function in the QPC. It agrees with the analytical model, which also predicts very strong thermal broadening for weak scanning gates.

While weak gates allow for high spatial resolution, strong gates maximize the position sensitivity, because small position changes can yield large variations in conductance. This is the case in the raising edges of a strong scanning gate and is widely exploited in scanning gate microscopy in the solid-state context [21]. To study this effect, we extract the derivative of conductance with position $d G / d x$, as shown in Fig. 4(b) [23]. The extremal variation rates mark the falling edges of the profiles and separate with increasing gate strength. The evolution of the width of the profile is clearly visible, as well as the clipping regime when the strong scanning gate is located at the center of the QPC. The fastest variations amount to $2.7(3) / h$ per micrometer.

The position sensitivity of our apparatus is limited by the signal to noise ratio with which we can measure conductances. The figure of merit is $\delta x=(d G / d x)^{-1} \delta G$, where $\delta G$ is the noise in the conductance measurement. To assess the minimal noise, we use the overlapping Allan deviation [45], giving $0.024(12) / h$ [46]. This translates into a position sensitivity of $\delta x=9(5) \mathrm{nm}$. The sensitivity characterizes our instrument and is mainly limited by the shotto-shot noise in the preparation of the reservoirs.
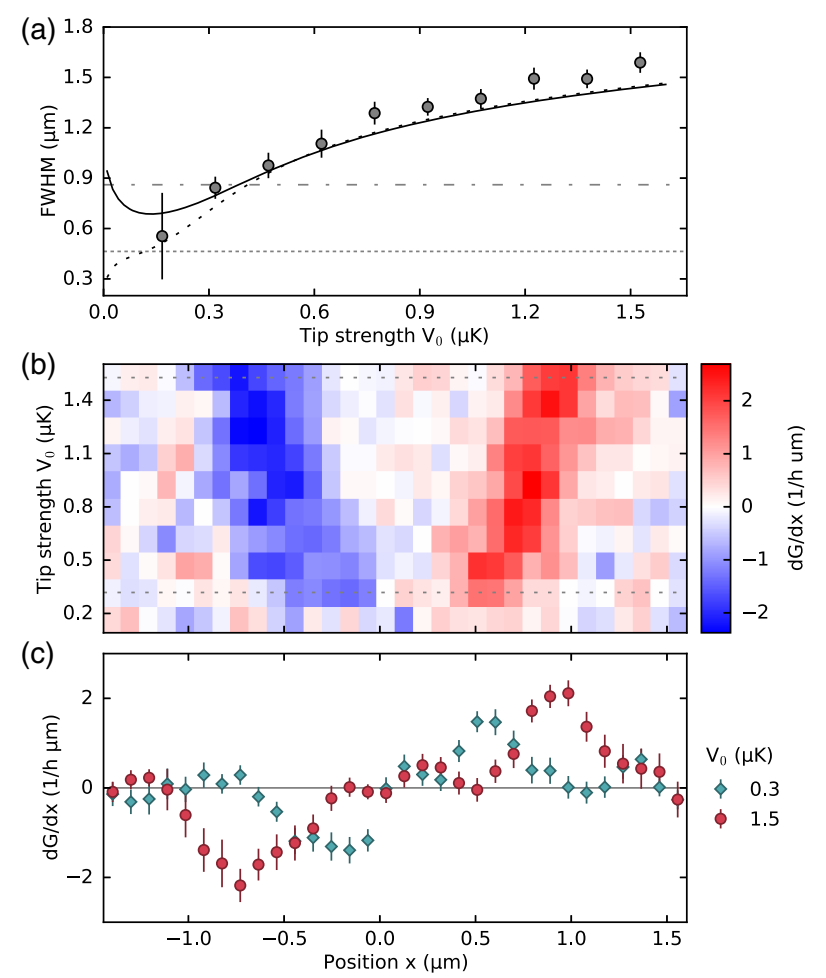

FIG. 4. Resolution and sensitivity of the scanning gate technique. (a) FWHM of the transverse scans through the QPC's center, shown in Fig. 3, as a function of the tip strength. Beside the experimental points, the dashed and solid lines represent the predictions of the analytical model for zero and a finite temperature of 58(5) nK. For a comparison, the FWHM of the obstacle in the transverse direction (dashed dotted line) and of the transverse probability distribution (horizontal dotted line), depicted in Fig. 3(a), are indicated. (b) Derivative of the measured conductance with respect to the position of the tip as a function of its position $x$ and its strength $V_{0}$. (c) Representative cuts along the dashed lines in (b). Conductance measurements are maximally sensitive to the position, for the strongest gate, where the derivative gets extremal.

In contrast to the condensed matter situation, our implementation can be generalized to several probes, where we expect interferences to strongly affect transport. In the many-body regime, these are known to have spectacular consequences such as pinning in a Luttinger liquid [47]. By further tailoring the probes, they may serve as building blocks for more complex atomtronics circuits [48-54]. Time-modulated or near-resonant optical gates that address external or internal atomic degrees of freedom could allow us to locally generate effective gauge structures. Such gates could also be used to perform spectroscopic measurements in analogy with scanning tunneling microscopy [55].

Our scanning gate technique can be generalized to any cold-atom system in which conductance measurements can be performed, such as disordered systems [56], yielding the additional ability to control the potential at a scale shorter than the localization length. It could distinguish percolation 
processes from localization by interferences or be combined with density measurements to identify the fraction of the atoms participating in transport [57]. In superfluid Fermi gases, the scanning gate could manipulate local modes like Andreev bound states [52,53]. It could also help to identify dynamical structures such as vortex patterns [58].

We thank A. Georges and T. Giamarchi for discussions and B. Bräm, L. Corman, R. Desbuquois, R. Steinacher, P. Törmä, D. Weinmann, and W. Zwerger for careful reading of the manuscript and discussions. We acknowledge financing from NCCR QSIT Contract No. 51NF40-160591, the ERC project SQMS Grant Agreement No. 247340, the FP7 project SIQS Grant Agreement No. 600645, the Horizon2020 project QUIC Grant Agreement No. 641122, and Swiss NSF under division II.S. N. acknowledges support from JSPS. J.-P. B. is supported by the Ambizione program of the Swiss NSF, by the Sandoz Family Foundation-Monique de Meuron program for Academic Promotion and by the ERC project DECCA.

* Corresponding author. samuehae@phys.ethz.ch

${ }^{\dagger}$ Corresponding author. esslinger@phys.ethz.ch *Corresponding author. jean-philippe.brantut@epfl.ch

[1] G. Binnig and H. Rohrer, Rev. Mod. Phys. 59, 615 (1987).

[2] F. J. Giessibl, Rev. Mod. Phys. 75, 949 (2003).

[3] M. A. Topinka, B. J. LeRoy, R. M. Westervelt, S. E. J. Shaw, R. Fleischmann, E. J. Heller, K. D. Maranowski, and A. C. Gossard, Nature (London) 410, 183 (2001).

[4] O. Fischer, M. Kugler, I. Maggio-Aprile, C. Berthod, and C. Renner, Rev. Mod. Phys. 79, 353 (2007).

[5] M. P. Allan et al., Nat. Phys. 11, 177 (2015).

[6] C.-C. Chien, S. Peotta, and M. Di Ventra, Nat. Phys. 11, 998 (2015).

[7] N. Gemelke, X. Zhang, C. Hung, and C. Chin, Nature (London) 460, 995 (2009).

[8] W. S. Bakr, J. I. Gillen, A. Peng, S. Fölling, and M. Greiner, Nature (London) 462, 74 (2009).

[9] J. F. Sherson, C. Weitenberg, M. Endres, M. Cheneau, I. Bloch, and S. Kuhr, Nature (London) 467, 68 (2010).

[10] M. F. Parsons, F. Huber, A. Mazurenko, C. S. Chiu, W. Setiawan, K. Wooley-Brown, S. Blatt, and M. Greiner, Phys. Rev. Lett. 114, 213002 (2015).

[11] L. W. Cheuk, M. A. Nichols, M. Okan, T. Gersdorf, V. V. Ramasesh, W. S. Bakr, T. Lompe, and M. W. Zwierlein, Phys. Rev. Lett. 114, 193001 (2015).

[12] E. Haller, J. Hudson, A. Kelly, D. A. Cotta, B. Peaudecerf, G. D. Bruce, and S. Kuhr, Nat. Phys. 11, 738 (2015).

[13] G. J. A. Edge, R. Anderson, D. Jervis, D. C. McKay, R. Day, S. Trotzky, and J. H. Thywissen, Phys. Rev. A 92, 063406 (2015).

[14] A. Omran, M. Boll, T. A. Hilker, K. Kleinlein, G. Salomon, I. Bloch, and C. Gross, Phys. Rev. Lett. 115, 263001 (2015).
[15] R. Yamamoto, J. Kobayashi, T. Kuno, K. Kato, and Y. Takahashi, New J. Phys. 18, 023016 (2016).

[16] E. Cocchi, L. A. Miller, J. H. Drewes, M. Koschorreck, D. Pertot, F. Brennecke, and M. Köhl, Phys. Rev. Lett. 116, 175301 (2016).

[17] T. Gericke, P. Wurtz, D. Reitz, T. Langen, and H. Ott, Nat. Phys. 4, 949 (2008).

[18] S. Krinner, D. Stadler, D. Husmann, J.-P. Brantut, and T. Esslinger, Nature (London) 517, 64 (2015).

[19] M. A. Eriksson, R. G. Beck, M. Topinka, J. A. Katine, R. M. Westervelt, K. L. Campman, and A. C. Gossard, Appl. Phys. Lett. 69, 671 (1996).

[20] M. A. Topinka, B. J. LeRoy, S. E. J. Shaw, E. J. Heller, R. M. Westervelt, K. D. Maranowski, and A. C. Gossard, Science 289, 2323 (2000).

[21] H. Sellier, B. Hackens, M. G. Pala, F. Martins, S. Baltazar, X. Wallart, L. Desplanque, V. Bayot, and S. Huant, Semicond. Sci. Technol. 26, 064008 (2011).

[22] Y. Imry and R. Landauer, Rev. Mod. Phys. 71, S306 (1999).

[23] See Supplemental Material at http://link.aps.org/ supplemental/10.1103/PhysRevLett.119.030403 for details, which includes Ref. [24-35].

[24] P. Zupancic, P. M. Preiss, R. Ma, A. Lukin, M. E. Tai, M. Rispoli, R. Islam, and M. Greiner, Opt. Express 24, 13881 (2016).

[25] J. E. Thomas, J. Kinast, and A. Turlapov, Phys. Rev. Lett. 95, 120402 (2005).

[26] M. J. H. Ku, A. T. Sommer, L. W. Cheuk, and M. W. Zwierlein, Science 335, 563 (2012).

[27] E. R. Sanchez Guajardo, M. K. Tey, L. A. Sidorenkov, and R. Grimm, Phys. Rev. A 87, 063601 (2013).

[28] L. D. Carr, G. V. Shlyapnikov, and Y. Castin, Phys. Rev. Lett. 92, 150404 (2004).

[29] G. Su, J. Chen, and L. Chen, Phys. Lett. A 315, 109 (2003).

[30] C. J. Pethick and H. Smith, Bose-Einstein Condensation in Dilute Gases (Cambridge University Press, Cambridge, England, 2002).

[31] D. W. Allan, Proc. IEEE 54, 221 (1966).

[32] F. Czerwinski, A. C. Richardson, and L. B. Oddershede, Opt. Express 17, 13255 (2009).

[33] D. A. Howe, D. U. Allan, and J. A. Barnes, in Proceedings of the Thirty-Fifth Annual Frequency Control Symposium, 1981 (IEEE, Piscataway, New Jersey, 1981), pp. 669-716, DOI: 10.1109/FREQ.1981.200541.

[34] E. C. Kemble, Phys. Rev. 48, 549 (1935).

[35] E. Hairer, S. P. Nørsett, and G. Wanner, Solving Ordinary Differential Equations I (Springer, New York, 1993).

[36] J.-P. Brantut, J. Meineke, D. Stadler, S. Krinner, and T. Esslinger, Science 337, 1069 (2012).

[37] C. Gorini, R. A. Jalabert, W. Szewc, S. Tomsovic, and D. Weinmann, Phys. Rev. B 88, 035406 (2013).

[38] S. Krinner, M. Lebrat, D. Husmann, C. Grenier, J.-P. Brantut, and T. Esslinger, Proc. Natl. Acad. Sci. U.S.A. 113, 8144 (2016).

[39] W. Szewc, theses, Université de Strasbourg, 2013.

[40] C. W. Groth, M. Wimmer, A. R. Akhmerov, and X. Waintal, New J. Phys. 16, 063065 (2014).

[41] L. I. Glazman, G. B. Lesovik, D. E. Khmel'Nitskiř, and R. I. Shekhter, Zh. Eksp. Teor. Fiz. Pis'ma Red. 48, 218 (1988). 
[42] T. Ihn, Semiconductor Nanostructures (Oxford University, New York, 2010).

[43] I. Affleck, Phys. Rev. Lett. 46, 388 (1981).

[44] L. Novotny and B. Hecht, Principles of Nano-Optics (Cambridge University Press, Cambridge, England, 2012).

[45] W. Riley and D. A. Howe, Handbook of Frequency Stability Analysis, NIST Special Publication 1065 (National Institute of Standards and Technology, Gaithersburg, MD, 2008).

[46] We performed 128 repeated conductance measurements in the single-mode regime without a scanning gate. The Allan deviation becomes minimal upon binning 29 consecutive points [23].

[47] C. L. Kane and M. P. A. Fisher, Phys. Rev. B 46, 15233 (1992).

[48] M. Albiez, R. Gati, J. Fölling, S. Hunsmann, M. Cristiani, and M. K. Oberthaler, Phys. Rev. Lett. 95, 010402 (2005).

[49] R. A. Pepino, J. Cooper, D. Z. Anderson, and M. J. Holland, Phys. Rev. Lett. 103, 140405 (2009).
[50] R. A. Pepino, J. Cooper, D. Meiser, D. Z. Anderson, and M. J. Holland, Phys. Rev. A 82, 013640 (2010).

[51] S. Eckel, J. G. Lee, F. Jendrzejewski, N. Murray, C. W. Clark, C. J. Lobb, W. D. Phillips, M. Edwards, and G. K. Campbell, Nature (London) 506, 200 (2014).

[52] D. Husmann, S. Uchino, S. Krinner, M. Lebrat, T. Giamarchi, T. Esslinger, and J.-P. Brantut, Science 350, 1498 (2015).

[53] G. Valtolina et al., Science 350, 1505 (2015).

[54] S. Eckel, J. G. Lee, F. Jendrzejewski, C. J. Lobb, G. K. Campbell, and W. T. Hill, Phys. Rev. A 93, 063619 (2016).

[55] A. Kantian, U. Schollwöck, and T. Giamarchi, Phys. Rev. Lett. 115, 165301 (2015).

[56] B. Shapiro, J. Phys. A 45, 143001 (2012).

[57] S. Krinner, D. Stadler, J. Meineke, J.-P. Brantut, and T. Esslinger, Phys. Rev. Lett. 115, 045302 (2015).

[58] M. Beria, Y. Iqbal, M. Di Ventra, and M. Müller, Phys. Rev. A 88, 043611 (2013). 